\title{
The Sound of Consciousness: Neural Underpinnings of Auditory Perception
}

\author{
Alfredo Brancucci, ${ }^{1}$ Raffaella Franciotti, ${ }^{2,3}$ Anita D'Anselmo, ${ }^{2}$ Stefania della Penna, ${ }^{2,3}$ and Luca Tommasi ${ }^{2}$ \\ Departments of ${ }^{1}$ Biomedical Sciences and ${ }^{2}$ Neuroscience and Imaging, ${ }^{3}$ Institute for Advanced Biomedical Technologies of the "G. d'Annunzio" University \\ Foundation, "G. d'Annunzio" University of Chieti and Pescara, 66100 Chieti Scalo, Italy
}

The neural correlates of consciousness (NCC), i.e., patterns of brain activity that specifically accompany a particular conscious experience, have been investigated mainly in the visual system using particularly suited paradigms, such as binocular rivalry and multistable percepts in combination with neural recordings or neuroimaging. Through the same principles, we look here for possible NCC in the auditory modality exploiting the properties of the Deutsch's illusion, a stimulation condition in which a sequence of two specular dichotic stimuli presented in alternation causes an illusory segregation of pitch and side (ear of origin), which can yield up to four different auditory percepts per dichotic stimulus. Using magnetoencephalography in humans, we observed cortical activity specifically accompanying conscious experience of pitch inside an early bilateral network, including the Heschl's gyrus, the middle temporal gyrus, the right inferior, and the superior frontal gyri. The conscious experience of perceived side was instead accompanied by later activity observed bilaterally in the inferior parietal lobe and in the superior frontal gyrus. These results suggest that the NCC are not independent of stimulus features and modality and that, even at the higher cortical levels, the different aspects of a single perceptual scene may not be simultaneously processed.

\section{Introduction}

Consciousness is a multifaceted feature that appears at different levels in biological organisms owning a complex nervous system. One of its aspects that are today more suitable to be investigated at a basic level is the neural activity related to a single conscious percept. In this view, a neural correlate of consciousness (NCC) is a precise pattern of brain activity that specifically accompanies a particular conscious experience.

It is actually not clear how any physical process, including neural activity, can give rise to a subjective phenomenon such as consciousness (Chalmers, 1996), but some promising empirical and theoretical headways have been made (Rees et al., 2002; Tononi, 2004; Tononi and Koch, 2008; Dehaene and Changeux, 2011; Edelman et al., 2011). The search for the NCC is thus an experimental subject that remains initially neutral on issues concerning causality, seeking instead to identify and characterize patterns of neural activity that specifically correlate with conscious experience and seeking to control for possible confounds

\footnotetext{
Received Aug. 2, 2011; revised Aug. 30, 2011; accepted Sept. 4, 2011.

Author contributions: A.B. designed research; A.B., R.F., and A.D. performed research; A.B., R.F., A.D., and S.d.P. analyzed data; A.B. and L.T. wrote the paper.

This research is part of the project Evolution and Development of Cognitive, Behavioral, and Neural Lateralization, 2006 -2010, supported by the Commission of the European Communities within the framework of the specific research and technological development program Integrating and Strengthening the European Research Area (initiative "What It Means to Be Human"), through a financial grant (L.T.). We thank Dr. Caterina Padulo for her technical support.

The authors declare no competing financial interests.

Correspondence should be addressed to Dr. Alfredo Brancucci, Department of Biomedical Sciences, "G. d'Annunzio" University of Chieti and Pescara, Via dei Vestini 31, 66100 Chieti Scalo, Italy. E-mail: alfredo.brancucci@unich.it.

DOI:10.1523/JNEUROSCI.3949-11.2011

Copyright $\odot 2011$ the authors $\quad 0270-6474 / 11 / 3116611-08 \$ 15.00 / 0$
}

using experimental designs that dissociate the contributions of consciousness from other (un)conscious cognitive functions (Frith et al., 1999). It could be debated whether attention should be included among these functions, because its status has historically divided scholars between those who equate it strictu sensu with consciousness (i.e., James, 1890) and those who separate the two phenomena (Koch and Tsuchiya, 2007).

Despite the relatively high number of studies investigating neural correlates of visual consciousness (Leopold and Logothetis, 1996; Blake and Logothetis, 2002), there is surprisingly very little evidence about the neural activity associated with auditory consciousness. The aim of the present study was to use an approach conceptually similar to the one of multistable visual percepts to explore consciousness in the auditory domain. In that approach, despite stimulus constancy, subjects experience diverse (typically two) conscious percepts. Correlating subjective report to the brain activations of the participant is a reliable method to explore the NCC (Tononi et al., 1998; Rees et al., 2002). To this aim, we chose the auditory sequence eliciting the Deutsch's illusion (also called "Octave illusion"; Deutsch, 1974), composed of a train of dichotic sine tones spaced an octave apart (typically 400 and $800 \mathrm{~Hz}$; Brancucci et al., 2009) presented repeatedly in alternation so that, when the right ear receives the high tone, the left ear receives simultaneously the low tone and vice versa. Subjects typically perceive an illusory single low-pitch tone at one ear alternating with an illusory single high-pitch tone at the other ear (see Fig. 1).

Central to the present study is that the stimulation is made up of two stimuli: a dichotic pair composed of a $400 \mathrm{~Hz}$ tone presented at the left ear and an $800 \mathrm{~Hz}$ tone presented at the right ear (here referred to as STIM A) and a specular dichotic pair composed of an 
$800 \mathrm{~Hz}$ tone presented at the left ear and a $400 \mathrm{~Hz}$ tone presented at the right ear (referred to as STIM B). The illusory single low-pitch tone alternating with the single high-pitch tone between ears are, however, not fixedly associated with one of the two dichotic pairs, nor between within subjects, which can perceive a single dichotic pair (e.g., STIM A) as having high or low pitch and at the left or at the right ear. However, it is noteworthy that, during one auditory sequence, the association between stimulus and percept does not change (Deutsch, 1974).

We used magnetoencephalography (MEG) to observe brain responses to each dichotic pair constituting the auditory stimulations and to relate them to the subjective report of the participants (Brancucci and Tommasi, 2011). The hypothesis is that lower-level auditory areas, but frontoparietal areas in particular, will show a neural activity strongly associated with the subjective report of the participants, similar to what found for visual consciousness (Rees et al., 2002).

\section{Materials and Methods}

Subjects. Nineteen subjects ( 13 females, six males) aged between 21 and 31 years (mean age, 25.1 years) participated in the study. None of them had auditory impairments as measured by auditory functional assessment (absolute hearing threshold $<20 \mathrm{~dB}$ ), and no differences $( \pm 5 \mathrm{~dB}$ ) of hearing threshold were found between the left and right ear (Brancucci et al., 2005). Subjects were recruited on the basis of their percept in the Deutsch's illusion assessed in a preliminary experiment to represent as much as possible all perception variants of the stimulation during the MEG recordings (i.e., low vs high pitch and left vs right ear for each stimulus). Therefore, both right-handers and left-handers were recruited, because it has been described that the percepts reported during the illusion are related to hand preference (Deutsch, 1983). Handedness measurement (Salmaso and Longoni, 1985) yielded 54.8 as an average result at group level, with three subjects scoring $<0$.

Stimuli. Stimuli were synthesized on a personal computer by means of the CSound language for sound synthesis (Vercoe, 1992). All tones were sinusoids having a duration of $500 \mathrm{~ms}$ and a frequency of 400 or $800 \mathrm{~Hz}$. Amplitude envelope presented an attack of $10 \mathrm{~ms}$ and a decay of $490 \mathrm{~ms}$. Tones were arranged in the sequence that constitutes the stimulation condition of the study (Fig. 1, top). The sequence is composed of the 400 and $800 \mathrm{~Hz}$ tones that compose two dichotic pairs $(400 \mathrm{~Hz}$ left, $800 \mathrm{~Hz}$ right: STIM A; $800 \mathrm{~Hz}$ left, $400 \mathrm{~Hz}$ right: STIM B). The pairs are presented repeatedly in alternation without interstimulus interval so that, when the right ear receives the high tone, the left ear receives the low tone and vice versa. The tone sequence, starting with STIM A, lasted $15 \mathrm{~s}$ and was presented 25 times with an interval of $8 \mathrm{~s}$ between two sequences. Additional 25 sequences starting with STIM B were presented. Stimulation intensity was $70 \mathrm{dBA}$. The acoustic stimulation was provided by Sensorcom plastic ear tubes connected to a transducer that was placed inside a $\mu$-metal box to avoid any artifact on the recordings. The initial stimulus of the sequence has been counterbalanced within subjects $(25+25$ sequences), whereas the position of the earpieces has been counterbalanced between subjects (i.e., to control for possible output differences in the earpieces, half of the subjects wore them in inverted position). Audio files of the stimuli can be downloaded (see Notes).

During each sequence, subjects had to press a button to indicate when they started to perceive the alternation between a low-pitch tone at one ear and a high-pitch tone at the other ear typical of the Deutsch's illusion. Previous works have shown that the percept during the Deutsch's octave illusion changes at every tone and consists of a single tone, having the same duration of the stimulus, that alternates between ears and assumes alternately a low and a high pitch (Deutsch, 1974; Zwicker, 1984). The average time necessary for the starting of the illusory percept (high-low alternation between ears) was $2.55 \pm 0.46 \mathrm{~s}$.

In addition, at the end of each sequence, subjects had to press one of four buttons describing the subjective properties of the last perceived tone: (1) low tone at the left ear, (2) high tone at the left ear, (3) low tone at the right ear, and (4) high tone at the right ear. The task allowed us to know the subjective illusory percepts associated with the two stimuli, which can vary both within subjects (between sequences) and between subjects. Of note, within one single sequence, the association between stimulus and percept does not change (Rees et al., 2002). The present paradigm should minimize possible confounds between attention and consciousness, a central issue in the search of the NCC (Koch and Tsuchiya, 2007), because the percept during the Deutsch's illusion depends in no way on attentional shifts of the subject (Rees et al., 2002).

$M E G$ recordings. The magnetic field was recorded by using a wholehead MEG system consisting of 165 dc SQUID integrated magnetometers and located inside a good quality magnetically shielded room (Della Penna et al., 2000). Evoked magnetic fields were bandpass filtered at $0.16-250 \mathrm{~Hz}$ and recorded at $1 \mathrm{kHz}$ sampling rate. To determine the position of the subject's head with respect to the sensor, the magnetic field generated by five coils placed on the scalp was recorded before and after each measurement session. A coordinate system was defined by digitizing anatomical landmarks on the subject's head by a 3D digitizer (3Space Fastrak; Polhemus). Cardiac and ocular activities were also monitored by means of bipolar electrode placed on the chest and on periorbital region to filter out possible heart and eyes contaminations of the MEG signals. Coregistration of MEG and magnetic resonance images of subjects' heads were obtained by means of a Philips scanner at $1.5 \mathrm{~T}$ using 3D T1-turbo field echo sequence. During the acquisition, spherical oil capsules were applied on the anatomical landmarks to allow coregistration of MEG and MRI coordinate systems. Then, 3D anatomical images were transformed into stereotaxic coordinates of the space of Talairach.

Data analysis. The experiment has been designed to obtain an average response from each brain site during the different percepts elicited by the same stimulus (STIM A or B).

As a first step, MEG segments corresponding to the four possible percepts (low tone at the left ear, high tone at the left ear, low tone at the right ear, high tone at the right ear) were separated on the basis of the subjective report starting from the last perceived tone, indicated by the second button press, and going backward until the starting of the illusion, indi- 
cated by the first button press. For example, if at the end of a sequence the subject pressed the button corresponding to "high tone at the right ear" (which indicates the last tone of the sequence he perceived), then the last, the third last, the fifth, seventh, ninth, etc. from the last MEG segments until the start of the illusory percept were considered as the perceptual condition "high-right," and automatically the second last, the fourth, sixth, eighth, etc. from the last MEG segments until the start of the illusory percept were considered as the perceptual condition "low-left," according to the well-known perceptual rules of the Deutsch's illusion. This was similar for the two other possible subjective perceptions.

As a second step, the perception categories along each dimension (i.e., pitch and ear of perception) were associated with the physical stimuli eliciting them. Thus, we obtained MEG trials in which the pitch was perceived as "high" or "low" and in which the sound was perceived at the left or right ear that were associated with STIM A $(400 \mathrm{~Hz}$ at the left ear and $800 \mathrm{~Hz}$ at the right ear) and MEG trials in which the pitch was perceived as "high" or "low" and in which the sound was perceived at the left or at the right ear that were associated with STIM B $(800 \mathrm{~Hz}$ at the left ear and $400 \mathrm{~Hz}$ at the right ear). Evoked magnetic fields were averaged from -50 to $+450 \mathrm{~ms}$ with stimulus onset at $0 \mathrm{~ms}$. The amplitude of the evoked fields was calculated with respect to a baseline level chosen as the mean value of the interval from -50 to $0 \mathrm{~ms}$ (of note, an additional analysis showed that the choice of a baseline period from 0 to $50 \mathrm{~ms}$ produced negligible differences in the results). To identify generators of MEG-evoked responses, different approaches are possible. They are divided into parametric methods assuming point-like sources as models (Scherg and Berg, 1996) and imaging methods such as minimum-norm estimates, low-resolution tomographies, and beam-forming approaches (Hämäläinen and Ilmoniemi, 1994; Pascual-Marqui et al., 2002, Sekihara et al., 2002; Brookes et al., 2007). Here an imaging approach was adopted because no a priori assumption on the number of sources could be driven from data. Among the imaging methods, we selected LORETA (lowresolution brain electromagnetic tomography; Pascual-Marqui et al., 2002) that can provide a blurred image of a point source centered on the location of maximal activity, even for shallow and correlated sources. Using the BESA software, the activity at each voxel of the $3 \mathrm{D}$ volume grid having a spacing of $7 \mathrm{~mm}$ was estimated for each time point. The areas of interest were chosen on the basis of a statistical analysis revealing the most active sites in the poststimulus interval. Such analysis was performed on each voxel by comparing source amplitudes in the poststimulus period (from 0 to $450 \mathrm{~ms}$ ) with the amplitude in the baseline period (from -50 to $0 \mathrm{~ms}$ ). $z$-scored values were obtained for each subject with the null hypothesis that the activity was the same all over the brain. Grand average of $z$-scores across subjects were thresholded at $p=0.001$ (Bonferroni corrected) on the basis of the number of MEG channels. If more than one voxel showed significant activity in the same area (Brodmann boundaries), only the most active one has been further considered. Indeed, the peak voxel obtained by LORETA should correspond to the possible location of the source. According to this analysis, the selected voxels were inside the inferior parietal lobe (IPL; BA39; Talairach coordinates, $\pm 37,-49,26$ ), the Heschl's gyrus (HG; BA41; $\pm 55,-19,13$ ), the middle temporal gyrus (MTG; BA21; $\pm 51,-3,-18$ ), the inferior frontal gyrus (IFG; BA13; $\pm 43,20,8$ ), the middle frontal gyrus (MFG; BA10; $\pm 27,48,3$ ), and the superior frontal gyrus (SFG; BA8; $\pm 17,35$, 41). Finally, on the basis of the literature on consciousness, according to which the NCC is comprised in an interval ranging from $\sim 100$ to $400 \mathrm{~ms}$ after stimulus onset in both vision (Pins and Ffytche, 2003; Wilenius and Revonsuo, 2007; Kinsey et al., 2009; Melloni et al., 2011) and audition (Maekinen et al., 2004; Gutschalk et al., 2008; for review, see Dehaene and Changeux, 2011; Railo et al., 2011) and strictly on the basis of the temporal shape of the present MEG responses (Fig. 2), mean MEG subjective responses were analyzed within the following epochs: 80-130, 130-300, and 300-450 in the HG, IPL, and IFG, and 100-300 and 300-450 in the MTG, MFG, and SFG (Fig. 2).

As a third step, we compared mean LORETA traces (each epoch) associated with either STIM A or STIM B in the voxels showing the highest level of activation in the poststimulus intervals described previously. Specifically, within traces evoked by STIM A, the comparisons were made between those associated with the perception of the high
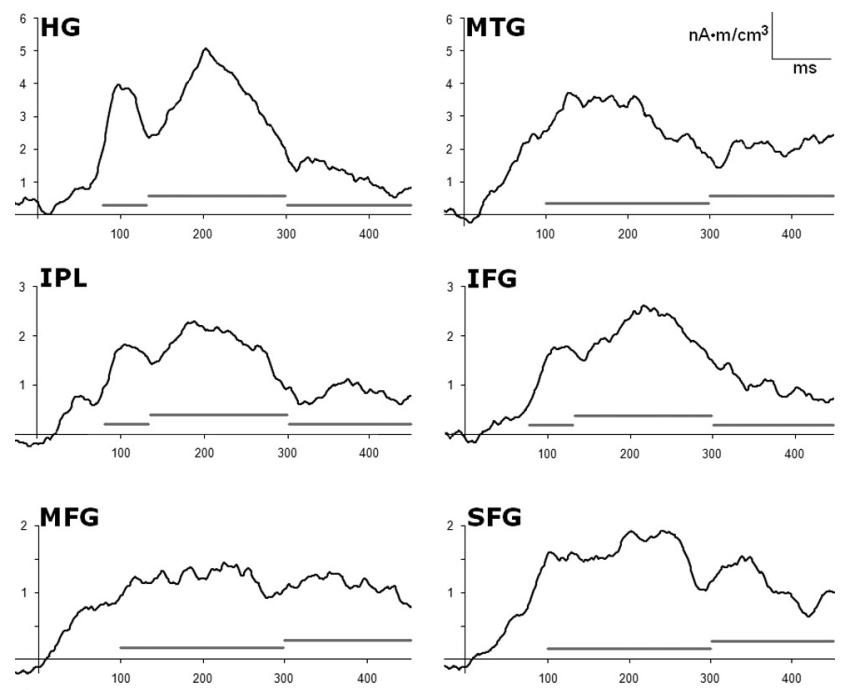

Figure 2. Temporal profile of LORETA responses. Group mean evoked responses (all data) in the most active voxel within the six areas showing strongest activity in the interval $0-450 \mathrm{~ms}$ after stimulus. Gray bars indicate temporal segmentation for statistical analyses (80-130, $130-300$, and $300-450$ ms for HG, IFG, and IPL; $100-300$ and 300 - 450 ms for MTG, MFG, and SFG).

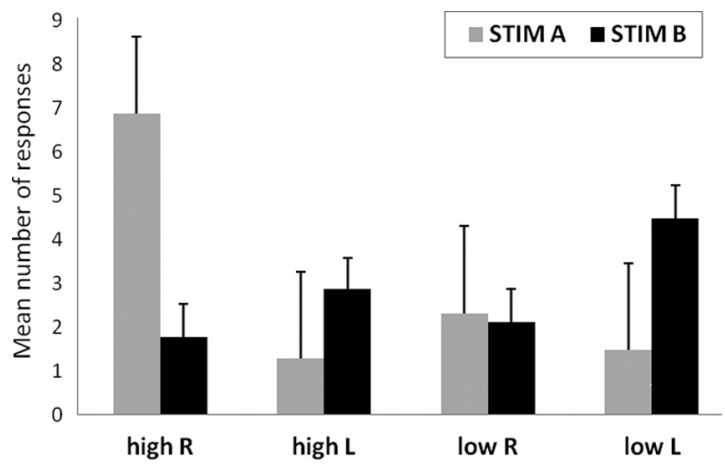

Figure 3. Behavioral results. The graph shows the distribution of the mean number of responses given by the subjects at the end of the sequences indicating the last perceived tone (high tone perceived at the right or left ear, low tone perceived at the right or left ear), separated for stimulus type (STIM A: $400 \mathrm{~Hz}$ left, $800 \mathrm{~Hz}$ right; STIM B: $800 \mathrm{~Hz}$ left, $400 \mathrm{~Hz}$ right). Error bars denote SEM.

versus low tone and, in a separate analysis, between those associated with a tone perceived at the left versus right ear. Similar comparisons were made within traces evoked by STIM B. Statistical analyses were performed on the values showed by the most active voxel within each area. The comparisons have been made by means of two repeated-measures ANOVAs with three factors: hemisphere (left, right), stimulus (STIM A, STIM B), and percept (high, low or left, right in separate analyses).

Of note, the number of trials used for the statistical comparisons was always balanced across perceptual conditions and within stimuli by rejecting the last trials of the sequences in which the more common percepts (STIM A, high percept and right percept; STIM B, low percept and left percept) were elicited, according to behavioral results.

\section{Results}

\section{Behavioral responses}

Figure 3 shows the distribution of the mean number of responses given by the subjects at the end of the auditory sequences indicating the last perceived tone (high tone perceived at the right or left ear, low tone perceived at the right or left ear) separated for stimulus type (STIM A: $400 \mathrm{~Hz}$ left, $800 \mathrm{~Hz}$ right; STIM B: $800 \mathrm{~Hz}$ left, $400 \mathrm{~Hz}$ right). When the sequence ended with STIM A, the mean number of responses was $8.11 \pm 1.67$ for high, $3.21 \pm 1.65$ 
for low and $9.79 \pm 1.56$ for right, $2.53 \pm 1.69$ for left perception. When the sequence ended with STIM B, the mean number of responses was $5.21 \pm 0.82$ for high, $6.95 \pm 0.79$ for low and $3.87 \pm 0.80$ for right, $7.79 \pm 0.77$ for left perception. ANOVA [ $2 \times 2 \times 2$, with STIM (A, B), perceived pitch (high, low), and perceived side (left, right) as factors] showed no main effects. All two-ways interactions were significant $($ STIM $\times$ perceived pitch, $F=6.98, p=0.017$; STIM $\times$ perceived side, $F=6.98, p<0.001$; perceived pitch $\times$ perceived side, $F=4.88, p=0.04)$. Three-way interaction was not significant. Significant post hoc tests (Duncan's test) were observed only for the STIM $\times$ perceived side interaction, in which with STIM A more right than left ear percepts were elicited $(p<0.001)$ and with STIM B more left than right ear percepts were elicited $(p<0.001)$.

In a separate experiment on another group of 19 participants recruited according to the same rules used for the subjects that underwent the MEG recordings concerning hearing threshold and perception of the Deutsch's illusion and matched with them for sex and age, we measured and compared the level of confidence in the responses given for pitch and ear of origin. Subjects sat in a chair on a table in front of the experimenter and were presented with auditory sequences having the same parameters as the ones presented during the MEG recordings (see Materials and Methods, Stimuli). Each sequence was presented four times, two of them with reversed earphones so that two sequences started with STIM A and two with STIM B. The task of the subjects was to report the pitch and the ear of origin of the last perceived tone of each sequence and to judge their confidence level for both responses on a Likert scale ranging from 1 (low confidence) to 7 (high confidence). Results showed that mean confidence levels were $5.42 \pm 0.22$ for pitch and $5.56 \pm 0.24$ for ear of origin. According to Student's $t$ test, the confidence levels in the two dimensions are not statistically different (two-tailed $t=0.72 ; p=$ 0.48). This result indicates that the following effects on the NCC of pitch and ear of origin are not biased by confidence.

\section{Neuromagnetic responses}

The brain voxels showing the strongest responses $(50-450 \mathrm{~ms}$ after stimulus) during the illusory percepts $(p<0.001)$ were located bilaterally in the following areas: IPL (BA39), HG (BA41), MTG (BA21), IFG (BA13), MFG (BA10), and SFG (BA8). Within these areas, ANOVA has been performed considering the factors hemisphere (left, right), stimulus (STIM A, STIM B), and percept (high, low or left, right in separate analyses: perceptual dimension of pitch and perceptual dimension of side). Of note, in such an experimental design, the search of the NCC is linked to the effects involving the factor percept. No main effect of percept has been observed. Conversely, concerning the high versus low perceptual dimension, an interaction of stimulus $\times$ percept was observed in the HG (80-130 ms after stimulus; $p=0.025)$, MTG (100-300 ms; $p=0.030)$, SFG $(100-300 \mathrm{~ms} ; p=0.034)$, and IFG (80-130 ms; $p=0.024$, triple interaction involving also the factor hemisphere). Concerning the left versus right perceptual dimension the same interaction was observed in the IPL $(300-450 \mathrm{~ms} ; p=0.012)$ and in the SFG $(300-450$ $\mathrm{ms} ; p=0.025)$. Figure 4 gives a sketch of the functional neuroanatomy involved in conscious perception observed in the present study by means of the LORETA difference maps from one representative subject. The colored areas in the maps represent the brain regions that behaved differently when the subjective perception of the same dichotic pair differed in terms of pitch (high vs low) or side (ear of origin, left vs right).

The group average activation values related to the significant interactions stimulus $\times$ percept are depicted graphically in Fig-
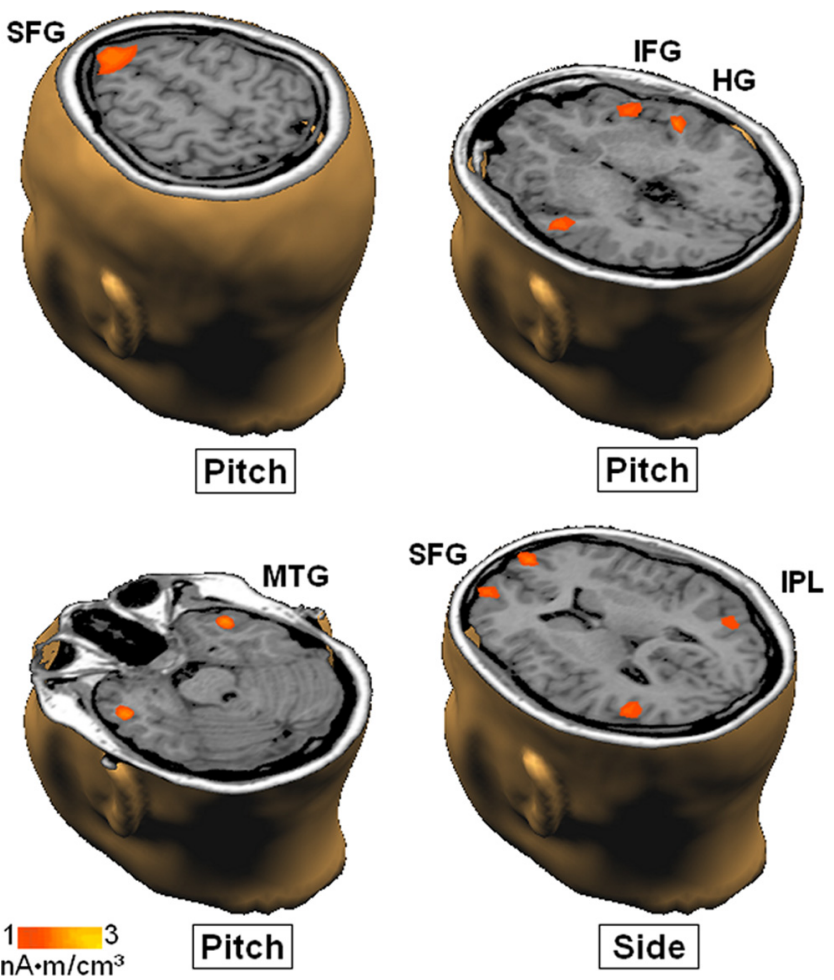

Figure 4. Difference maps from one representative subject. The brain regions that behaved differently when the subjective perception of the same dichotic pair differed in terms of pitch (first 3 maps) or side (ear of origin, bottom right) are colored. The maps referring to pitch have been calculated by subtracting the activity measured during the perception of a low-pitch tone from the activity observed during the perception of a high-pitch tone both elicited by STIM A. The map referring to side has been calculated by subtracting the activity measured during the perception of a tone in the right ear from the activity observed during the perception of a tone in the left ear, both elicited by STIM A.

ure 5. Concerning the perception of pitch, illustrated in the four graphs on the left, it can be observed that the conscious experience of an high tone during the stimulation with STIM A was associated with a stronger bilateral activation in the HG (in the time interval from 80 to $130 \mathrm{~ms}$ after stimulus), as well as in the MTG and SFG (100-300 ms) compared with the conscious experience of a low tone elicited by the same stimulus. In the IFG ( $80-130 \mathrm{~ms})$, such activation was asymmetrical, in that the right but not the left IFG showed a stronger activation in association with the perception of an high-pitch tone. During stimulation with STIM B, the associated activations were in general reversed but reached post hoc significance only in the MTG (stronger activation for low-pitch perception) and IFG, in which, although in the left hemisphere no relations with the conscious report were observed, in the right hemisphere the perception of a low-pitch tone was associated with stronger activity compared with the perception of an high-pitch tone.

Concerning perception of side (ear of origin), the two graphs on the right of Figure 5 show that, during the stimulation with STIM A, the perception of a tone in the left ear was associated with a stronger activation in both IPL (300-450 ms) and SFG (300-450 ms), whereas the stimulation with STIM B yielded an inverted activation pattern, associated with nonsignificant post hoc tests. It can be noticed that the stimulus $\times$ percept interactions involving the dimension of pitch occurred always between 80 and $300 \mathrm{~ms}$ poststimulus onset, whereas the ones involving side (ear of origin) occurred later, after $300 \mathrm{~ms}$ poststimulus onset. 


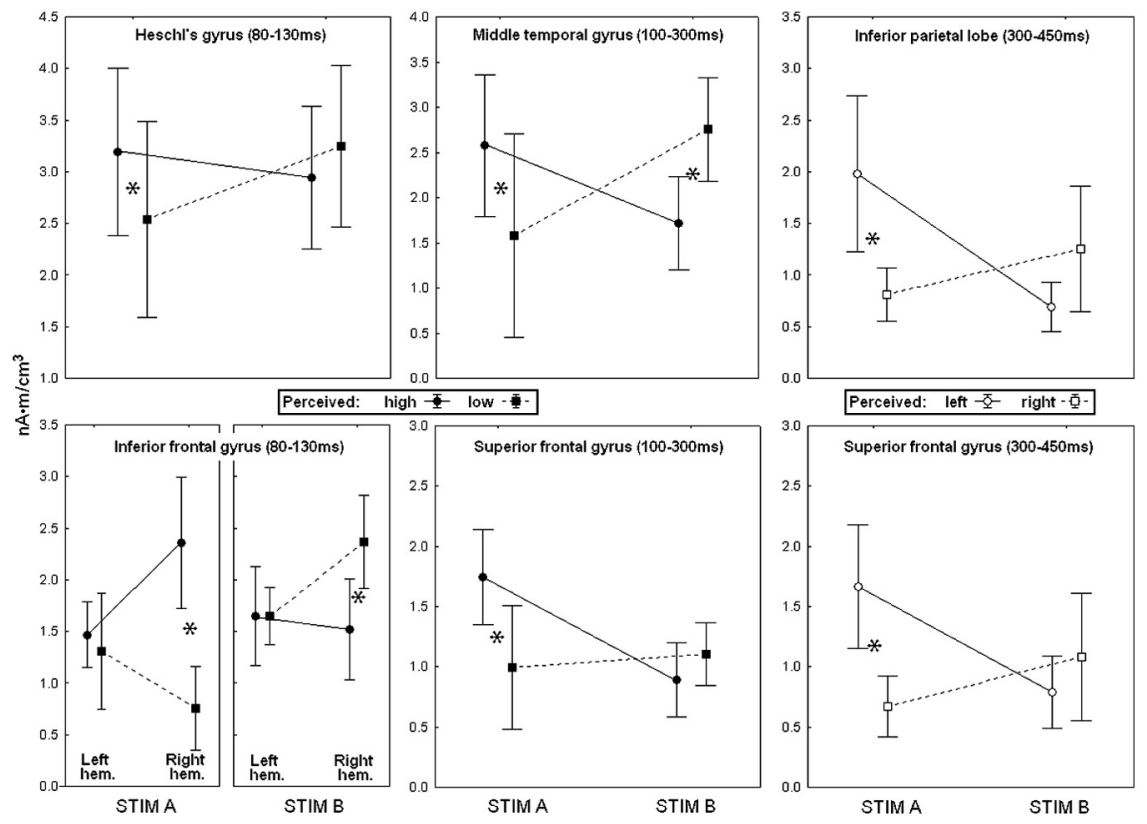

Figure 5. Mean results. Four left panels, Pitch perception. Two right panels, Side perception (ear of origin). Average brain response amplitude in the areas and time intervals in which the interaction stimulus $\times$ perception was significant (in the IFG, the interaction involved also the hemisphere). STIM A, $400 \mathrm{~Hz}$ at the left ear $+800 \mathrm{~Hz}$ at the right; STIM B, $800 \mathrm{~Hz}$ at the left ear +400 $\mathrm{Hz}$ at the right. ${ }^{*} p<0.05$, significant post hoc comparisons. Error bars denote SEM.

Table 1 lists all statistically significant results obtained. Because the aim of the present study was to catch differences in neural responses that are distinctively related to conscious perception, the relevant statistically significant results (described above) are emphasized in bold. Summarizing the other, minor significant results reported in Table 1 (those in normal font), it can be noticed that there is a general association between STIM B and the left hemisphere (i.e., stronger activation in the left hemisphere when STIM B is presented) in the IPL and MFG. Conversely, in the IPL and SFG, the opposite association is present, i.e., between STIM A and the right hemisphere. In addition, the IFG and SFG host, respectively, an association between the low percept and STIM B and between the high percept and STIM A. Moreover, in the right IPL and right SFG, STIM A produces a stronger activation than STIM B, and finally, during the perception of the sound in the left ear, STIM A produces a stronger activation than STIM B in the IPL and SFG. Globally, these results indicate that there is a link between STIM A, right hemisphere, and the high percept and between STIM B, left hemisphere, and the low percept, which involves primarily the IPL, IFG, MFG, and SFG.

\section{Discussion}

The present results showed that it is possible to observe a change in neural activity that is exclusively related to a change in a conscious auditory percept. This was obtained with the present experimental design by exploiting the perceptual features of the Deutsch's illusion in which the same auditory stimulus can elicit two different percepts on two dimensions, i.e., pitch, a high or a low tone, and side, a tone perceived at the left or right ear.

Associating brain activity with the subjective report, we found that the brain areas specifically processing the percept were not the same in the two perceptual dimensions. The pitch percept was specifically associated with symmetric activity in the HG, MTG, and SFG, as well as in the right IFG, whereas the side/ear of origin percept was specifically associated with symmetric activity in the IPL and SFG. These findings suggest that the neural networks subtending conscious experience are strictly related to conscious experience itself and confirm that there is not a single area that is responsible for consciousness (Crick and Koch, 2005; Tononi and Koch, 2008). Moreover, the lack of a main effect Percept suggests that, even if the mentioned areas behave in a different manner depending on subjects' conscious experience, their activation is at least in part stimulus driven, which means that there do not exist pure "areas of consciousness" completely released from the stimulus.

The cortical areas here observed to be involved in pitch and side perception overlap to a good extent the areas constituting, respectively, the auditory "what" and "where" pathways. These two auditory streams have been described by works performed mainly in the past 15 years using direct neural recordings in monkeys or neuroimaging techniques in humans (Rauschecker and Tian, 2000; Arnott et al., 2004; Woods and Alain, 2009). The "what" pathway projects from the anterior primary auditory cortex to more anterior areas, such as the planum polare (Ahveninen et al., 2006), the right IFG and the insulae (Renier et al., 2009) and then to the ventrolateral prefrontal cortex (Rauschecker and Scott, 2009). In turn, the "where" pathway projects from the posterior primary auditory cortex dorsally to more medioposterior areas, such as the planum temporale (Ahveninen et al., 2006), the IPL and the superior parietal lobe (Brunetti et al., 2005; Renier et al., 2009), and then to the dorsolateral prefrontal cortex (Brunetti et al., 2008; Rauschecker and Scott, 2009). What is noticeable in the present study is that these areas were activated by stimuli that bore no differences in spatial or pitch cues because they were physically identical. This indicates that the two auditory streams can be activated also by internal cues and that they maintain their specificity during top-down processing strategies.

An interesting observation is that, at the level of NCC, we found no lateralized processes related to side perception, that is, there was no enhanced activity in the (superior) cortical areas contralateral to the localization of the percept, as happens for lower-stages areas (Pantev et al., 1986). An additional intriguing result can be deduced from the time intervals in which the neural activity was specifically related to conscious experience. It can be noticed that the activations related to the quality of the percept (high vs low pitch) were earlier than those related to the ear of origin (left vs right) in which the sounds have been perceived, the first occurring $\sim 200 \mathrm{~ms}$ before the second. Thus, even at the higher cortical levels, the different aspects of a single perceptual scene are processed in series, despite the subjective feeling of plain simultaneity (Zeki, 2003).

The majority of the cortical areas we found to be specifically involved in the formation of auditory percepts have been associated previously with diverse aspects related to consciousness. The two anterior frontal gyri (IFG and SFG) have been frequently associated with consciousness as parts of the prefrontal cortex (Lumer et al., 1998; Tong et al., 2006). In addition, the IFG has been shown to be specifically involved in the emergence of consciousness of thirst (Egan et al., 2003) and in baseline brain activ- 
Table 1. Statistical results

\begin{tabular}{|c|c|c|c|c|c|c|}
\hline $\begin{array}{l}\text { Area (Talairach } \\
\text { coordinates, } x, y, z)\end{array}$ & $\begin{array}{l}\text { Time interval (ms } \\
\text { poststimulus onset) }\end{array}$ & Significant effects & $F$ & $p$ & Post hoc comparisons & $\begin{array}{l}p \\
\text { (Duncan's test) }\end{array}$ \\
\hline \multicolumn{7}{|c|}{ Perceptual dimension: pitch, high versus low } \\
\hline \multirow[t]{4}{*}{$\operatorname{IPL}( \pm 37,-49,24)$} & $80-130$ & & & & & \\
\hline & $130-300$ & & & & & \\
\hline & $300-450$ & Hemisphere $\times$ stimulus & 10.228 & 0.006 & STIM B: left hemisphere > right hemisphere & 0.041 \\
\hline & & & & & Right hemisphere: STIM A > STIM B & 0.005 \\
\hline \multirow[t]{3}{*}{$\mathrm{HG}( \pm 52,-19,17)$} & $80-130$ & Stimulus $\times$ percept & 6.120 & 0.025 & STIM A: high percept > low percept & 0.038 \\
\hline & $130-300$ & & & & & \\
\hline & $300-450$ & & & & & \\
\hline \multirow[t]{2}{*}{$\operatorname{MTG}( \pm 46,-3,-18)$} & $100-300$ & Stimulus $\times$ percept & 5.868 & 0.030 & STIM A: high percept > low percept & 0.027 \\
\hline & $300-450$ & & & & and STIM B: low percept > high percept & 0.036 \\
\hline \multirow[t]{4}{*}{$\mathrm{IFG}( \pm 43,20,8)$} & $80-130$ & Stimulus $\times$ percept & 5.545 & 0.033 & STIM A: high percept > low percept & 0.049 \\
\hline & $130-300$ & & & & Low percept: STIM B $>$ STIM A & 0.036 \\
\hline & $300-450$ & Hemisphere $\times$ stimulus $\times$ & 6.321 & 0.024 & Right hemisphere, STIM A: high percept > low percept & 0.001 \\
\hline & & percept & & & Right hemisphere, STIM B: low percept $>$ high percept & 0.009 \\
\hline \multirow[t]{2}{*}{ MFG $( \pm 27,45,3)$} & $100-300$ & Hemisphere $\times$ stimulus & 5.942 & 0.030 & STIM B: left hemisphere > right hemisphere & 0.035 \\
\hline & $300-450$ & & & & & \\
\hline \multirow[t]{2}{*}{$\operatorname{SFG}( \pm 17,35,41)$} & $100-300$ & Stimulus $\times$ percept & 5.443 & 0.034 & STIM A: high percept > low percept & 0.028 \\
\hline & $300-450$ & & & & and High percept: STIM A > STIM B & 0.016 \\
\hline \multicolumn{7}{|c|}{ Perceptual dimension: side (ear of orgin), left vs right } \\
\hline \multirow[t]{7}{*}{ IPL } & $80-130$ & Hemisphere $\times$ stimulus & 4.946 & 0.045 & Right hemisphere: STIM A > STIM B & 0.049 \\
\hline & $130-300$ & & & & & \\
\hline & $300-450$ & Hemisphere $\times$ stimulus & 15.965 & 0.002 & Right hemisphere: STIM A > STIM B & 0.002 \\
\hline & & & & & STIM B: left hemisphere $>$ right hemisphere & 0.009 \\
\hline & & & & & STIM A: right hemisphere $>$ left hemisphere & 0.035 \\
\hline & & Stimulus $\times$ percept & 8.543 & 0.012 & STIM A: left percept > right percept & 0.019 \\
\hline & & & & & Left percept: STIM A > STIM B & 0.013 \\
\hline \multirow[t]{3}{*}{ HG } & $80-130$ & & & & & \\
\hline & $130-300$ & & & & & \\
\hline & $300-450$ & & & & & \\
\hline \multirow[t]{2}{*}{ MTG } & $100-300$ & Hemisphere $\times$ percept & 5.205 & 0.040 & & \\
\hline & $300-450$ & Stimulus $\times$ percept & 5.182 & 0.040 & & \\
\hline \multirow[t]{3}{*}{ IFG } & $80-130$ & & & & & \\
\hline & $130-300$ & & & & & \\
\hline & $300-450$ & Percept (left > right) & 7.027 & 0.020 & & \\
\hline \multirow[t]{2}{*}{ MFG } & $100-300$ & Hemisphere $\times$ stimulus & 5.348 & 0.038 & & \\
\hline & $300-450$ & Hemisphere (left > right) & 6.313 & 0.026 & & \\
\hline \multirow[t]{5}{*}{ SFG } & $100-300$ & Hemisphere $\times$ stimulus & 4.906 & 0.045 & Right hemisphere: STIM A > STIM B & 0.046 \\
\hline & $300-450$ & Hemisphere $\times$ stimulus & 5.498 & 0.036 & Right hemisphere: STIM A > STIM B & 0.023 \\
\hline & & & & & STIM A: right hemisphere > left hemisphere & 0.032 \\
\hline & & Stimulus $\times$ percept & 6.418 & 0.025 & STIM A: left percept > right percept & 0.022 \\
\hline & & & & & Left percept: STIM A > STIM B & 0.036 \\
\hline
\end{tabular}

The table reports all significant results observed in the ANOVA performed for each selected area and time interval in the two perceptual dimensions (pitch, high vs low; ear/side, left vs right). The relevant statistically significant results are in bold.

ity during conscious rest (Mazoyer et al., 2001). The SFG is specifically involved in conscious self-regulation of emotions (Beauregard et al., 2001) and during conscious rest (Mazoyer et al., 2001). The candidacy of the IPL as a cortical area involved in the NCC is also fully underwritten by neuroscientists. Ten years ago, Taylor (2001) identified "the inferior parietal lobes as the best candidates for the NCC." This claim still agrees with a series of convergences that range over neglect, visual extinction, attention, and working memory (Smith and Jonides, 1997; Corbetta and Shulman, 2002; Ciçek et al., 2007), pointing to a strong implication of the IPL in awareness. More recently, the IPL has been implied also in self-awareness (Lou et al., 2004) and in the formation of percepts starting from especially complex stimuli (Sarri et al., 2006). Although a frontoparietal network of areas has been often observed in (visual) consciousness studies (Pins and Ffytche, 2003; Tononi and Koch, 2008), in the present results, the parietal cortex showed no differential activations during the perception of high and low pitch. However, concerning auditory perception, the involvement of parietal areas has been associated mostly with spatial aspects of perception rather than with other features, such as, broadly, identification (Rauschecker and Tian, 2000; Arnott et al., 2004; Woods and Alain, 2009). This interpretation is further supported by a study comparing frontoparietal activity in visual and auditory awareness (Eriksson et al., 2007). It showed that, although frontal regions were related to both visual and auditory awareness, parietal activity was related only to visual awareness and superior temporal activity was correlated with auditory awareness. According to the authors' interpretation, these results indicate that frontal regions interact with specific posterior regions to produce awareness in different sensory modalities. Conversely, a specific involvement of the MTG in the NCC has not been observed previously. The MTG is considered to be part of the inferotemporal cortex whose functions range from visual perception to language/semantic memory processing and multimodal sensory integration (Mesulam, 1998). The present findings point to an involvement of this area in auditory consciousness, possibly as an auditory-specific NCC. Finally, the role of the HG (containing the primary auditory 
cortex) in consciousness is a matter of debate. In the present study, we found a direct involvement of the HG in consciousness but only in relation to pitch perception. Previous studies suggested that primary auditory areas could be involved in some aspects of auditory consciousness (Bekinschtein et al., 2009; Bidet-Caulet and Bertrand, 2009). This would be a noticeable difference compared with visual consciousness in which there is now general agreement that the primary visual areas do not have a specific role in the NCC (Rees et al., 2002; Tononi and Koch, 2008). Future studies should deepen this subject, but such a dissimilarity between the visual and the auditory system would be not implausible given the intrinsic differences between the two modalities and maybe also given the spatial properties of the primary cortices. The visual cortex, which is much larger, lies spatially in a posterior possible "NCC periphery" compared with the auditory cortex, which occupies a small location between likely NCC candidates, such as inferotemporal cortex, insula, speech areas, and inferior parietal and prefrontal cortices.

In conclusion, the present study suggests that the NCC may depend at least in part on stimulus modality because some cortical areas that have been found to play a role in visual consciousness, such as the inferior temporal cortex, have not been observed here as part of the auditory NCC, whereas other areas, such as the MTG, have never been associated with the visual NCC, but here it has been observed to be strictly associated with the auditory NCC. Furthermore, still within one sensory modality, the NCC may involve quite different sets of areas, as suggested by the different networks observed here for pitch and side perception. Finally, even at the higher cortical levels, in which perception is presumed to be a highly integrated whole, at least some aspects of a single perceptual scene are still processed in series (Zeki, 2003).

\section{Notes}

Supplemental material for this article is available at http://www.ch. unich.it/facolta/psicologia/edcbnl/units/? $\mathrm{p}=$ dir $=$ chieti,page $=$ res. Audio files of the stimuli presented to the subjects. This material has not been peer reviewed.

\section{References}

Ahveninen J, Jääskeläinen IP, Raij T, Bonmassar G, Devore S, Hämäläinen M, Levänen S, Lin FH, Sams M, Shinn-Cunningham BG, Witzel T, Belliveau JW (2006) Task-modulated "what" and "where" pathways in human auditory cortex. Proc Natl Acad Sci U S A 103:14608-14613.

Arnott SR, Binns MA, Grady CL, Alain C (2004) Assessing the auditory dual-pathway model in humans. Neuroimage 22:401-408.

Beauregard M, Lévesque J, Bourgouin P (2001) Neural correlates of conscious self-regulation of emotion. J Neurosci 21:RC165(1-6).

Bekinschtein TA, Dehaene S, Rohaut B, Tadel F, Cohen L, Naccache L (2009) Neural signature of the conscious processing of auditory regularities. Proc Natl Acad Sci U S A 106:1672-1677.

Bidet-Caulet A, Bertrand O (2009) Neurophysiological mechanisms involved in auditory perceptual organization. Front Neurosci 3:182-191.

Blake R, Logothetis NK (2002) Visual competition. Nat Rev Neurosci 3:13-21.

Brancucci A, Tommasi L (2011) "Binaural rivalry": dichotic listening as a tool for the investigation of the neural correlate of consciousness. Brain Cogn 76:218-224.

Brancucci A, Babiloni C, Rossini PM, Romani GL (2005) Right hemisphere specialization for intensity discrimination of musical and speech sounds. Neuropsychologia 43:1916-1923.

Brancucci A, Padulo C, Tommasi L (2009) “Octave illusion" or "Deutsch's illusion"? Psychol Res 73:303-307.

Brookes MJ, Stevenson CM, Barnes GR, Hillebrand A, Simpson MI, Francis ST, Morris PG (2007) Beamformer reconstruction of correlated sources using a modified source model. Neuroimage 34:1454-1465.

Brunetti M, Belardinelli P, Caulo M, Del Gratta C, Della Penna S, Ferretti A, Lucci G, Moretti A, Pizzella V, Tartaro A, Torquati K, Olivetti Belardinelli
M, Romani GL (2005) Human brain activation during passive listening of sounds from different locations: a study with fMRI and MEG. Hum Brain Mapp 26:251-261.

Brunetti M, Della Penna S, Ferretti A, Del Gratta C, Cianflone F, Belardinelli P, Caulo M, Pizzella V, Olivetti Belardinelli M, Romani GL (2008) A fronto-parietal network for the spatial attention reorienting in the auditory domain: a human $\mathrm{fMRI} / \mathrm{MEG}$ study of functional and temporal dynamics. Cereb Cortex 18:1139-1147.

Chalmers D-J (1996) The conscious mind: in search of a fundamental theory. Oxford: Oxford UP.

Ciçek M, Gitelman D, Hurley RS, Nobre A, Mesulam M (2007) Anatomical physiology of spatial extinction. Cereb Cortex 17:2892-2898.

Corbetta M, Shulman GL (2002) Control of goal-directed and stimulusdriven attention in the brain. Nat Rev Neurosci 3:201-215.

Crick FC, Koch C (2005) What is the function of the claustrum? Philos Trans R Soc Lond B Biol Sci 360:1271-1279.

Dehaene S, Changeux JP (2011) Experimental and theoretical approaches to conscious processing. Neuron 70:200-227.

Della Penna S, Del Gratta C, Granata C, Pasquarelli A, Pizzella V, Rossi R, Russo M, Torquati K, Erne SN (2000) Biomagnetic systems for clinical use. Philos Mag B 80:937-948.

Deutsch D (1974) An auditory illusion. Nature 251:307-309.

Deutsch D (1983) The octave illusion in relation to handedness and familial handedness background. Neuropsychologia 21:289-293.

Edelman GM, Gally JA, Baars BJ (2011) Biology of consciousness. Front Psychol 2:4.

Egan G, Silk T, Zamarripa F, Williams J, Federico P, Cunnington R, Carabott L, Blair-West J, Shade R, McKinley M, Farrell M, Lancaster J, Jackson G, Fox P, Denton D (2003) Neural correlates of the emergence of consciousness of thirst. Proc Natl Acad Sci U S A 100:15241-15246.

Eriksson J, Larsson A, Ahlström KR, Nyberg L (2007) Similar frontal and distinct posterior cortical regions mediate visual and auditory perceptual awareness. Cereb Cortex 17:760-765.

Frith C, Perry R, Lumer E (1999) The neural correlates of conscious experience: an experimental framework. Trends Cogn Sci 3:105-114.

Gutschalk A, Micheyl C, Oxenham AJ (2008) Neural correlates of auditory perceptual awareness under informational masking. PLoS Biol 6:e138.

Hämäläinen MS, Ilmoniemi RJ (1994) Interpreting magnetic fields of the brain: minimum norm estimates. Med Biol Eng Comput 32:35-42.

James W (1890) The principles of psychology. New York: Holt.

Kinsey K, Anderson SJ, Hadjipapas A, Nevado A, Hillebrand A, Holliday IE (2009) Cortical oscillatory activity associated with the perception of illusory and real visual contours. Int J Psychophysiol 73:265-272.

Koch C, Tsuchiya N (2007) Attention and consciousness: two distinct brain processes. Trends Cogn Sci 11:16-22.

Leopold DA, Logothetis NK (1996) Activity changes in early visual cortex reflect monkeys' percepts during binocular rivalry. Nature 379:549-553.

Lou HC, Luber B, Crupain M, Keenan JP, Nowak M, Kjaer TW, Sackeim HA, Lisanby SH (2004) Parietal cortex and representation of the mental self. Proc Natl Acad Sci U S A 101:6827-6832.

Lumer ED, Friston KJ, Rees G (1998) Neural correlates of perceptual rivalry in the human brain. Science 280:1930-1934.

Mäkinen V, May P, Tiitinen H (2004) Transient brain responses predict the temporal dynamics of sound detection in humans. Neuroimage 21:701-706

Mazoyer B, Zago L, Mellet E, Bricogne S, Etard O, Houdé O, Crivello F, Joliot M, Petit L, Tzourio-Mazoyer N (2001) Cortical networks for working memory and executive functions sustain the conscious resting state in man. Brain Res Bull 54:287-298.

Melloni L, Schwiedrzik CM, Müller N, Rodriguez E, Singer W (2011) Expectations change the signatures and timing of electrophysiological correlates of perceptual awareness. J Neurosci 31:1386-1396.

Mesulam MM (1998) From sensation to cognition. Brain 121:1013-1052.

Pantev C, Lütkenhöner B, Hoke M, Lehnertz K (1986) Comparison between simultaneously recorded auditory evoked magnetic fields and potentials elicited by ipsilateral, contralateral and binaural tone burst stimulation. Audiology 25:54-61.

Pascual-Marqui RD, Esslen M, Kochi K, Lehmann D (2002) Functional imaging with low-resolution brain electromagnetic tomography (LORETA): a review. Methods Find Exp Clin Pharmacol 24 [Suppl C]:91-95.

Pins D, Ffytche D (2003) The neural correlates of conscious vision. Cereb Cortex 13:461-474. 
Railo H, Koivisto M, Revonsuo A (2011) Tracking the processes behind conscious perception: a review of event-related potential correlates of visual consciousness. Conscious Cognit 20:972-983.

Rauschecker JP, Scott SK (2009) Maps and streams in the auditory cortex: nonhumanprimates illuminate human speech processing. Nat Neurosci $12: 718-724$

Rauschecker JP, Tian B (2000) Mechanisms and streams for processing of "what" and "where" in auditory cortex. Proc Natl Acad Sci U S A 97: $11800-11806$

Rees G, Kreiman G, Koch C (2002) Neural correlates of consciousness in humans. Nat Rev Neurosci 3:261-270.

Renier LA, Anurova I, De Volder AG, Carlson S, VanMeter J, Rauschecker JP (2009) Multisensory integration of sounds and vibrotactile stimuli in processing streams for "what" and "where." J Neurosci 29:10950-10960.

Salmaso D, Longoni AM (1985) Problems in the assessment of hand preference. Cortex 21:533-549.

Sarri M, Blankenburg F, Driver J (2006) Neural correlates of crossmodal visual-tactile extinction and of tactile awareness revealed by fMRI in a right-hemisphere stroke patient. Neuropsychologia 44:2398-2410.

Scherg M, Berg P (1996) New concepts of brain source imaging and localization. Electroencephalogr Clin Neurophysiol Suppl 46:127-137.

Sekihara K, Nagarajan SS, Poeppel D, Marantz A (2002) Performance of an MEG adaptive-beamformer technique in the presence of correlated neural activities: effects on signal intensity and time course estimates. IEEE Trans Biomed Eng 49:1534-1546.
Smith EE, Jonides J (1997) Working memory: a view from neuroimaging. Cognit Psychol 33:5-42.

Taylor JG (2001) The central role of the parietal lobes in consciousness. Conscious Cogn 10:379-417.

Tong F, Meng M, Blake R (2006) Neural bases of binocular rivalry. Trends Cogn Sci 10:502-511.

Tononi G (2004) An information integration theory of consciousness. BMC Neuroscience 5:42.

Tononi G, Koch C (2008) The neural correlates of consciousness: an update. Ann NY Acad Sci 1124:239-261.

Tononi G, Srinivasan R, Russell DP, Edelman GM (1998) Correlates of conscious perception by frequency-tagged neuromagnetic responses. Proc Natl Acad Sci U S A 95:3198-3203.

Vercoe BL (1992) A manual for the audio processing system and supporting programs with tutorials. Cambridge, MA: Massachusetts Institute of Technology Media Laboratories.

Wilenius ME, Revonsuo AT (2007) Timing of the earliest ERP correlate of visual awareness. Psychophysiology 44:703-710.

Woods DL, Alain C (2009) Functional imaging of human auditory cortex. Curr Opin Otolaryngol Head Neck Surg 17:407-411.

Zeki S (2003) The disunity of consciousness. Trends Cogn Sci 7:214-218.

Zwicker T (1984) Experiments on the dichotic octave illusion (in German). Acustica 55:128-136. 